\title{
Microstructural and Microchemical Analyses of Extracted Second-Phase Precipitates in Alpha-Annealed and Beta-Quenched Zircaloy-4
}

\author{
Ken R. Anderson and Ram Bajaj \\ Bechtel Marine Propulsion Corp. - Bettis Atomic Power Laboratory West Mifflin, PA 15122
}

Zircaloy-4 is used as fuel-element cladding in the nuclear-power generation industry. As part of an overall study of the fundamentals of Zircaloy-4 (Zr-4) (a hexagonal close pack $\mathrm{Zr}-1.5 \% \mathrm{Sn}, 0.25 \% \mathrm{Fe}$, $0.125 \% \mathrm{Cr}$ alloy) corrosion, a detailed investigation of the second-phase precipitates that enhance the corrosion resistance of Zircaloy-4 in pressurized water reactors was pursued. In order to better characterize the microstructure and microchemistry of the precipitates, they were extracted from Zircaloy-4 material by dissolving the parent matrix in an acid solution and collecting residue of intact precipitates. Source material used included alpha-annealed and late beta-quenched Zircaloy-4.

Small metal pieces of each material type were submerged in a solution of methanol and $20 \%$ perchloric acid. With the Zircaloy-4 metal as the anode and a surrounding platinum wire mesh as the cathode, the electrolytic dissolution was achieved with an applied current of $\sim 2-5$ A. The electrolytic action dissolved the Zr-based matrix material into solution and left the second-phase precipitates suspended in solution. The solution was centrifuged to settle the particles out to the bottom of the test tube. The top liquid was poured off and the remaining precipitate residue was then rinsed several times in methanol and collected on a filter paper for drying. A small portion of the powder from each extraction was stirred in methanol and collected onto separate carbon-coated copper grids (150 mesh) and analyzed by TEM.

The precipitates on grids and jet polished foils were loaded into double-tilt analytical holders and analyzed in either a Philips CM300 field-emission gun (FEG) scanning transmission electron microscope (STEM) operated at $300 \mathrm{kV}$ or an FEI Tecnai TF20 FEG STEM. Microchemical analysis was performed in STEM mode using an effective electron probe size of $\sim 10 \mathrm{~nm}$, with the X-ray signal collected using the attached thin-window Oxford detector interfaced with the Oxford INCA energy dispersive spectroscopy (EDS) analysis system. A minimum of 110 extracted Zr-Fe-Cr-bearing secondphase precipitates were analyzed for the two Zircaloy-4 heat treatment conditions. The electron beam was usually positioned approximately in the center of each precipitate.

The extraction allowed for the relative ease of direct analysis of second-phase precipitates without interference from the Zr-based matrix typically present in a foil sample. This allowed for more definitive microchemical, crystal structure and size data of the precipitates. Based on the analysis of a large number of precipitates in each material, the average measured iron-to-chromium (Fe/Cr) ratios (in at \%) for the alpha-annealed Zircaloy-4 was $2.06 \pm 0.10$ with a range of $\sim 1$ to 3.25 . The Laves precipitates had an average $\mathrm{Zr}$ content of $\sim 35 \mathrm{at} \%$ and an average $\mathrm{Fe}+\mathrm{Cr}$ value of $\sim 65$ at $\%$, which confirms the $\mathrm{AB}_{2}$-type formula, i.e. $\mathrm{Zr}(\mathrm{Fe}, \mathrm{Cr})_{2}$. Figure 1 shows a scatter plot of the $\mathrm{Fe} / \mathrm{Cr}$ data for alpha-annealed Zircaloy-4. Of the precipitates analyzed for the alpha-annealed Zircaloy-4, the Zr-Fe-Cr-containing precipitates were mostly the hexagonal $(\mathrm{C} 14)$ polymorph of the $\mathrm{Zr}(\mathrm{Fe}, \mathrm{Cr})_{2}$ Laves phase, with a small percentage $(<10 \%)$ being the cubic $(\mathrm{C} 15)$ polymorph, as based on electron diffraction analysis. (TEM images of precipitates extracted from the alpha-annealed $\mathrm{Zr}-4$ are shown in Figure 2). For the beta-quenched Zircaloy-4, the mixture of $\mathrm{C} 14$ and $\mathrm{C} 15$ was closer to 50/50. There was no direct correlation between crystal structure and $\mathrm{Fe} / \mathrm{Cr}$ ratios in the alpha-annealed and beta-quenched material. 
There was a distinct difference between the two materials in the second-phase precipitate morphology. The alpha-annealed $\mathrm{Zr}-4$ precipitates were oblong with a size range of from 0.2 to 0.4 microns and betaquenched material had precipitates that were narrow, long and "finger-like." Crystal structure and the morphology of the second-phase precipitates observed are likely factors in the corrosion behavior of Zircaloy-4.

With the extensive TEM/STEM analysis, this study was an expansion of previous, more limited, work on Zircaloy-4 [1]. From the current investigation it was shown that the analysis of the extracted particles provides a much clearer picture of the chemistry, crystal structure and the size / shape of the second phase particles in Zircaloy-4 than is possible from in-matrix analyses.

[1] C. Toffolon-Masclet, J-C. Brachet and G. Jago, J. Nuc. Mater. 305 (2002) 224.

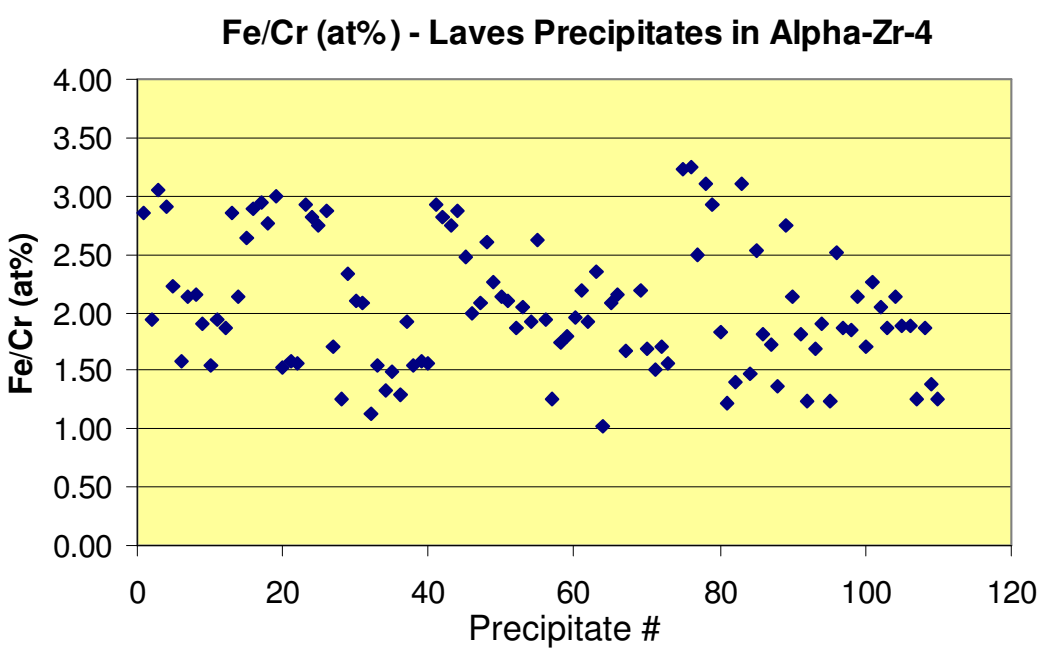

Figure 1. Fe/Cr (in at\%) scatter plot for Lavesphase precipitates in alpha-annealed $\mathrm{Zr}-4$.

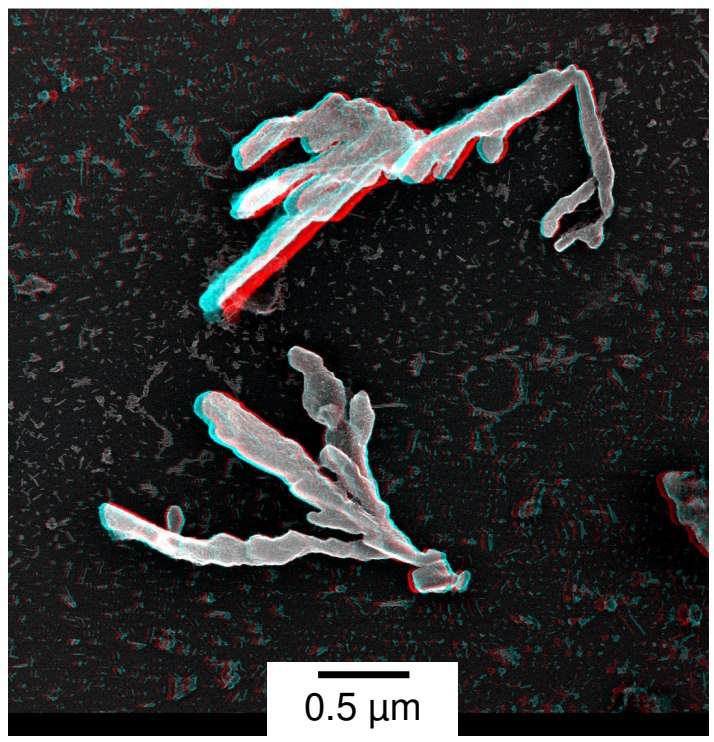

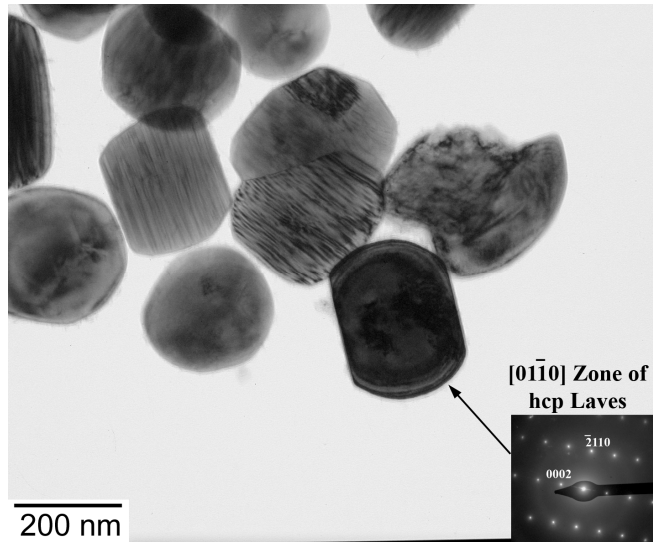

Figure 2. TEM image of Lavesphase precipitates extracted from alpha-annealed Zircaloy-4, with mostly hexagonal $(\mathrm{C} 14) \mathrm{Zr}(\mathrm{Fe}, \mathrm{Cr})_{2}$ Laves precipitates.

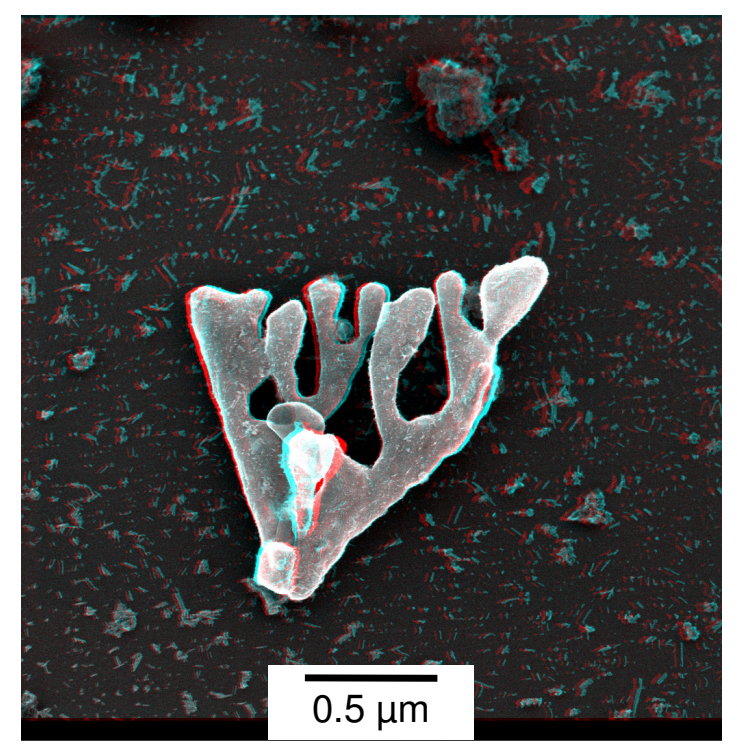

Figure 3. STEM images (using secondary electrons), as 3D anaglyphs, of Laves-phase precipitates from beta-treated Zr-4. 